\title{
Does Preoperative Computed Tomography Angiography Lead to an Increased Incidence of Postoperative Acute Kidney Injury Following Transcatheter Aortic Valve Implantation in Patients with Impaired Renal Function?
}

\author{
Dritan Useini $\mathrm{MD}^{1 *}$, Blerta Beluli $\mathrm{MD}^{2}$, Hildegard Christ Dr. rer. Medic. ${ }^{3}$, Zulfugar Taghiyev \\ MD ${ }^{1}$, Markus Schlömicher MD ${ }^{1}$, Mahmoud Elghannam MD ${ }^{1}$, Peter Haldenwang MD PhD ${ }^{1}$, \\ Matthias Bechtel MD PhD', Justus Strauch MD PhD ${ }^{1}$ \\ ${ }^{I}$ Department of Cardiothoracic Surgery, Ruhr-University Hospital Bergmannsheil, Bochum, Germany \\ ${ }^{2}$ Department of Internal Medicine, St. Anna Hospital, Herne, Germany \\ ${ }^{3}$ Institute of Medical Statistics and Computational Biology (IMSB), University Hospital of \\ Cologne, Germany
}

*Corresponding Author: Dritan Useini MD, Department of Cardiothoracic Surgery, Ruhr-University Hospital Bergmannsheil, Bochum, Germany, Email: dritan-83@live.com

\begin{abstract}
Aim: Although the superiority of information derived from computed tomography angiography (CTA) compared to transesophageal echocardiography (TOE) for aortic annulus sizing has been proved, the CTA is considered critically due to the contrast media (CM) application, especially in patients with compromised renal function. We aimed to evaluate the impact of CTA on the incidence of postoperative acute kidney injury (AKI) following transapical transcatheter aortic valve implantation (TA-TAVI).

Methods: Two hundred and thirteen high-risk patients with severe aortic stenosis who underwent TA-TAVI in our institution between 01/2011 and 08/2016, were analyzed. In conformity with our policy switch in 01/2014 from TOE to CTA for annulus sizing, the cohort was divided in two groups, (TOE-group/CTAgroup) and retrospectively analyzed. The occurrence of a post-procedural AKI was defined according to the VARC-2 criteria.

Results: The preoperative risk evaluation was similar in both groups (EuroSCORE II: $8.2 \pm 6.5 \%$ in CTA vs. $7.8 \pm 6.2 \%$ in TOE). Overall AKI occurred in 75 patients (35.2\%), with no group difference (33\% in CTA vs. $37.4 \%$ in TOE; $p=0,567)$. Preoperative chronic kidney disease (CKD) was seen in 97 patients (45.5\%). Here AKI occurrence was $32.4 \%$ in CTA vs. $39.3 \%$ in TOE; $p=0,888$. A post-procedural renal replacement therapy (RRT) was needed in 26 patients (12.2\%); the need was less in case of patients with CTA $(6.6 \%$ in CTA vs. $17.8 \%$ in TOE; $p=0,01$ ). Patients with pre-existing CKD needed RRT postoperatively in $15.6 \%$ in CTA, vs. $25.4 \%$ in TOE $(p<0,001)$.
\end{abstract}

Conclusion: The preoperative CTA is a safe investigation method prior to TA-TAVI and is not associated with an increased incidence of $A K I$, even in patients with $C K D$.

Keywords:computed tomography angiography, contrast media, TA-TAVI, postoperative acute kidney injury.

\section{INTRODUCTION}

A key factor for the success of transcatheter aortic valve implantation (TAVI) techniques is the accurate anatomical measurements of the aorta. Dimensions such as the aortic annulus diameter, perimeter, orifice area, and the distance to the coronary orifices or detailed analysis of calcification patterns have a major relevance not only to achieve good operative results, but also to avoid catastrophic, lifethreatening complications during TAVI

ARC Journal of Surgery procedures. The superiority of the computed tomography angiography (CTA) compared to transesophageal echocardiography (TOE) in terms of annulus sizing and post-procedural complications is well documented [1,2]. At this point, due to the advantages of CTA compared to TOE [3], CTA has become the standard method of evaluation in pre-TAVI procedures $[4,5]$.

On the other hand, CTA represents a source for kidney injury, due to need for contrast media 
Does Preoperative Computed Tomography Angiography Lead to an Increased Incidence of Postoperative Acute Kidney Injury Following Transcatheter Aortic Valve Implantation in Patients with Impaired Renal Function?

(CM) application. The TAVI population typically includes elderly patients with significant pre-existing renal dysfunction. The acute kidney injury (AKI) represents a severe complication following TAVI, which is known to increase the post-procedural morbidity and mortality [6-9]. We aimed to assess whether the preoperative CTA examination leads to an increased incidence of postoperative AKI after transapical (TA)-TAVI.

\section{METHODS}

Patients with aortic valve stenosis, deemed highrisk candidates for surgical aortic valve replacement were considered for TA-TAVI, according to our standardized interdisciplinary heart-team protocol. We retrospectively analyzed patients who underwent TA-TAVI in our institution between 01/2011 and 08/2016. Initially, in our TA-TAVI era, we preoperatively employed TOE (Philips CX50, Revision 3.1.1, Philips Ultrasound; Andover, MA, USA) for annulus sizing. Since 01/2014, in conformity to our policy switch, almost all TA-TAVI candidates preoperatively underwent CTA for annulus sizing. We performed CTA using a mono-source CT machine (Somatom Definition Edge, Simens, Germany; 128 detector rows, rapid ECG gated, spiral acquisition, covering the heart and the thoracic aorta) $24 \mathrm{~h}$ before TATAVI, with intravenous application of $60 \mathrm{ml}$ CM (370 mg iodine/ml; Solutrast 370; Bracco Imaging, Milan, Italy). A CM volume bolus was injected at the rate of $4 \mathrm{ml} / \mathrm{s}$ followed by a crystalloid solution of $40 \mathrm{ml}$. The imaging parameters for the reference tube current and voltage were set to $160-190 \mathrm{mAs}$ and $120 \mathrm{kVp}$, respectively. The image acquisition was performed in cranio-caudal scan direction. The applied CM volume was the same in all patients $(60 \mathrm{ml})$. All patients gave written informed consent before the CTA examination. We did not use a standardized hydration regime. All patients intraoperatively underwent intra-arterial CM exposure (370 mg iodine/ml; Solutrast 370; fluoroscopy performance. For the patient group with CTA, there was a second CM exposure within $24 \mathrm{~h}$. This patient cohort intraoperatively received for fluoroscopy on average $69 \pm 20 \mathrm{ml}$ of $\mathrm{CM}$ in contrast to the TOE which received 77 $\pm 27 \mathrm{ml}$ of $\mathrm{CM}$ for fluoroscopy [Fig.5].We performed a CTA examination by all patients, independently of chronic kidney disease (CKD) co morbidity. We defined CKD here as Stage 3 or 4 according to renal dysfunction, injury to the kidney, failure or loss of kidney function, and end-stage kidney disease (RIFLE) classification. So we were able to divide the cohort into two groups: 107 (50. 2\%) patients who received TOE and 106 (49. 8\%) who received CTA preoperatively. After the exclusion of patients with chronic dialysis, 213 patients were included in this study. The postoperative AKI was defined according to Valve Academic Research Consortium Consensus Document (VARC-2) criteria. Ethics Committee approval has been obtained (Reg. Nr.18-6339).

\section{Statistical ANAlysis}

For continuous variables, data are reported as mean with standard deviation. The categorical variables are reported as frequency (percentage). Data comparison was done using the $\chi^{2}$ test, the Fisher's exact test, Kruskal-Wallis test and the Mann-Whitney test as appropriate. All statistical tests were 2 -sided, and $p$ values of 0.05 or less were considered statistically significant. The statistical analyses were conducted using SPSS 23.0.0.2 software (IBM, Chicago, IL, USA).

\section{ReSUlts}

A total of 229 patients ( $84 \pm 7$ years, $53.5 \%$ male, EuroSCORE II $8 \pm 6 \%$ ) who underwent TATAVI in our institution were analyzed. After excluding patients with chronic dialysis, 213 patients were included in this study. Preoperatively, for annulus sizing, 107 patients underwent TOE $(50.2 \%)$ and 106 CTA $(49.8 \%)$. Preoperative variables of the entire cohort and of the two subgroups, CTA and TOE, are shown in Table1.

Bracco Imaging, Milan, Italy) during

Table1: Baseline clinical characteristics (* left ventricular ejection fraction)

\begin{tabular}{|l|l|l|l|l|}
\hline Table 1 & All & TOE & CTA & \\
& $\mathbf{N = 2 1 3 ;} \mathbf{n =}(\boldsymbol{\%})$ & $\mathbf{N = 1 0 7} ; \mathbf{n = ( \% )}$ & $\mathbf{N = 1 0 6 ; n = ( \% )}$ & $\boldsymbol{p}$ Value \\
\hline & & & & \\
\hline Age & $84 \pm 7$ & $86 \pm 6$ & $82 \pm 6$ & $p<0,0001$ \\
\hline Male sex & $114(53,5)$ & $58(54,2)$ & $56(52,3)$ & $p=0,677$ \\
\hline EuroScore II & $8 \pm 6,32$ & $7,81 \pm 6,22$ & $8,23 \pm 6,47$ & $p=0,703$ \\
\hline Arterial Hypertension & $189(88,7)$ & $99(92,5)$ & $90(84,9)$ & $p=0,117$ \\
\hline Diabetes mellitus & $73(34,2)$ & $37(34,5)$ & $36(33,9)$ & $p=1$ \\
\hline
\end{tabular}


Does Preoperative Computed Tomography Angiography Lead to an Increased Incidence of Postoperative Acute Kidney Injury Following Transcatheter Aortic Valve Implantation in Patients with Impaired Renal Function?

\begin{tabular}{|c|c|c|c|c|}
\hline LVEF* & $49 \pm 11$ & $47 \pm 10$ & $51 \pm 11$ & $p=0,001$ \\
\hline Peripheral artery disease & & & & \\
\hline Chronic kidney failure & & & & \\
\hline Preoperative creatinine $(\mathrm{mg} / \mathrm{dL})$ & $3 \pm$ & $1,4=$ & $1,2 \pm$ & 0,0001 \\
\hline Atrial fibril & & & & $=0,002$ \\
\hline Previous cardiac operation & $37(17,4)$ & $13(12,1)$ & $24(22,6)$ & $p=0,03$ \\
\hline \multicolumn{5}{|c|}{ 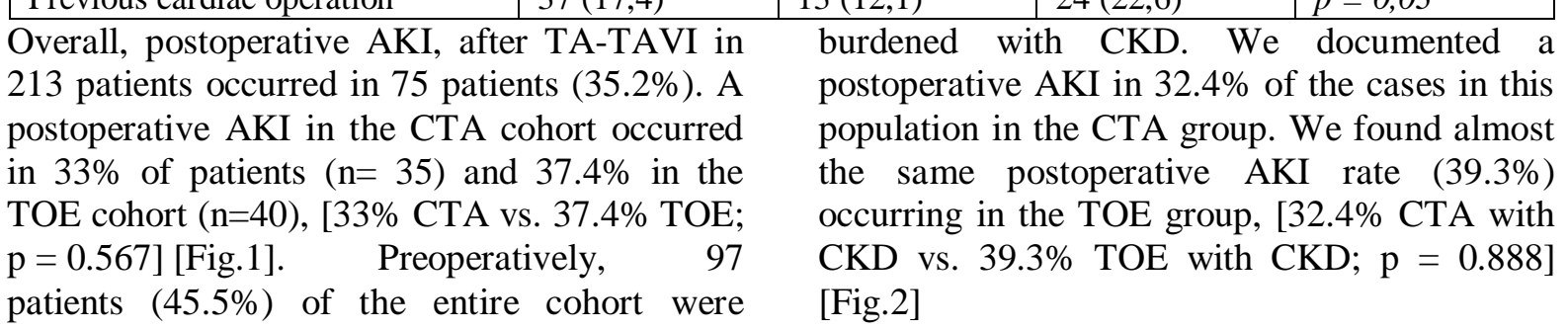 } \\
\hline
\end{tabular}
patients $(45.5 \%)$ of the entire cohort were [Fig.2]

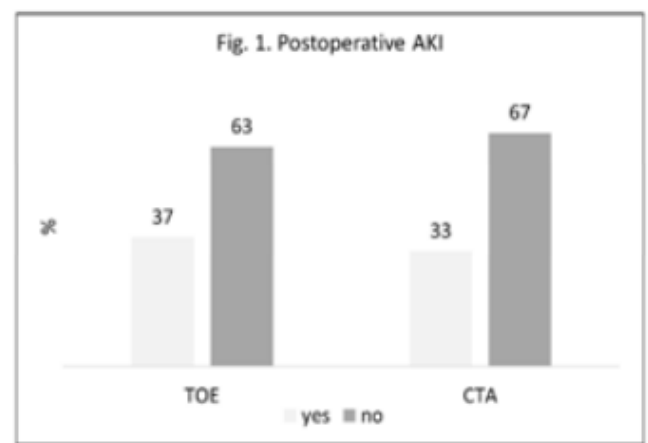

Fig1: Comparison of the postoperative acute kidney injury (AKI) occurrence among patients who received CTA and TOE preoperatively for annulus sizing.

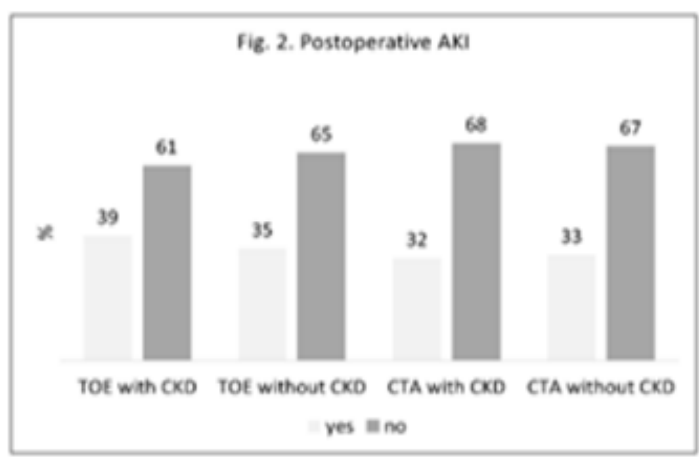

Fig2: Comparison of the postoperative acute kidney injury (AKI) occurrence among CTA and TOE groups of patients with chronic kidney disease (CKD).

From a total of 213 patients, postoperative renal replacement therapy (RRT) was necessary in 26 patients $(12.2 \%)$. In the CTA group, we registered RRT in $6.6 \%$ of the cases, in the TOE group in $17.8 \%$ of the cases, [6.6\% CTA vs.
$17.8 \%$ TOE; $\mathrm{p}=0.01]$. We also documented a significantly more RRT in the TOE group compared to the CTA group, in the patients with CKD, $[15.6 \%$ CTA with CKD vs. $25.4 \%$ TOE with CKD; $\mathrm{p}<0.001]$ [Fig.3].

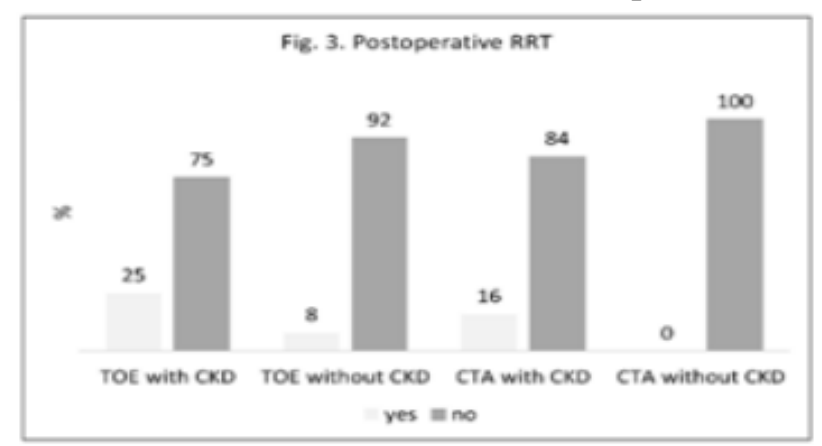

Fig3: Comparison of patients who needed renal replacement therapy (RRT) postoperatively in CTA and TOE groups with and without chronic kidney disease (CKD). 
Does Preoperative Computed Tomography Angiography Lead to an Increased Incidence of Postoperative Acute Kidney Injury Following Transcatheter Aortic Valve Implantation in Patients with Impaired Renal Function?

The intraoperative $\mathrm{CM}$ application during fluoroscopy was $73 \pm 24 \mathrm{ml}$ in the entire cohort and $69 \pm 20 \mathrm{ml} / 77 \pm 27 \mathrm{ml}$ in the CTA / TOE group respectively $(\mathrm{p}=0.015)$. The perioperative total $\mathrm{CM}$ volume applied was significantly higher in the CTA group (CTA + fluoroscopy) compared to the TOE group (fluoroscopy), $[129 \pm 20 \mathrm{ml} \mathrm{CTA}$ vs. $77 \pm 27 \mathrm{ml}$ TOE; $\mathrm{p}<0.001$ ] [Fig.4].The 30-day- all-causemortality of the entire cohort was $10.1 \%$. Owing to postoperative AKI-related complications 3 patients died from the TOE group but none from the CTA group. Further perioperative characteristics are shown in Table 2, 3 .

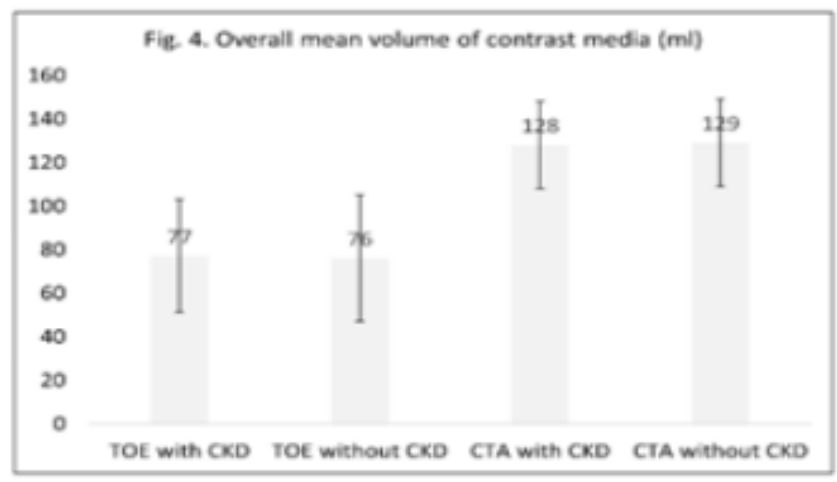

Fig4: Periprocedural applied total volume contrast media in both CTA and TOE groups.

Table2: Procedural and postprocedural characteristics (* cardio-pulmonary bypass)

\begin{tabular}{|c|c|c|c|c|}
\hline Table 2 & $\begin{array}{l}\text { All } \\
\mathrm{N}=213 ; n=(\%)\end{array}$ & $\begin{array}{l}\text { TOE } \\
\mathbf{N}=107 ; n=(\%)\end{array}$ & $\begin{array}{l}\text { CTA } \\
\mathrm{N}=106 ; \mathrm{n}=(\%)\end{array}$ & $p$ Value \\
\hline TOE-derived annulus diameter (mm) & I & $23 \pm 2,1$ & / & / \\
\hline CTA-derived annulus diameter (mm) & / & I & $24,2 \pm 2,5$ & I \\
\hline Prosthesis diameter $(\mathrm{mm})$ & $25,5 \pm 2,1$ & $25,57 \pm 2,1$ & $25,45 \pm 2,1$ & $p=1$ \\
\hline Contrast medium application (mL) & $103 \pm 41$ & $77 \pm 27$ & $129 \pm 20$ & $p<0,001$ \\
\hline Mean procedural time (h/min) & $01: 47 \pm 00: 44$ & $01: 53 \pm 00: 55$ & $01: 40 \pm 00: 24$ & $p=0,354$ \\
\hline Conversion to $\mathrm{CPB}^{*}$ & $9(4,2)$ & $7(6,5)$ & $2(1,9)$ & $p=0,178$ \\
\hline Bleeding $(\mathrm{mL})$ & $465 \pm 305$ & $502 \pm 338$ & $420 \pm 256$ & $p=0,08$ \\
\hline AKI-related-30-day-mortality & $3(1,4)$ & $3(2,8)$ & $0(0)$ & $p=0,260$ \\
\hline
\end{tabular}

Table3: AKI-related post-procedural characteristics $(*$ acute kidney injury; $* *$ renal replacement therapy (continuous veno-venous hemodialysis))

\begin{tabular}{|l|l|l|l|l|}
\hline Table 3 & All & TOE & CTA & \\
& $\mathbf{N = 2 1 3 ; n = ( \% )}$ & $\mathbf{N = 1 0 7 ; n = ( \% )}$ & $\mathbf{N = 1 0 6 ; n = ( \% )}$ & $\boldsymbol{p}$ Value \\
\hline Postprocedural AKI* & $75(35,2)$ & $40(37,4)$ & $35(33,0)$ & $p=0,567$ \\
\hline AKI Stage 1 & $48(64)$ & $27(67,5)$ & $21(60)$ & $p=0,507$ \\
\hline AKI Stage 2 & $20(26,7)$ & $11(27,5)$ & $9(25,7)$ & $p=0,863$ \\
\hline AKI Stage 3 & $7(9,3)$ & $2(5)$ & $5(14,3)$ & $p=0,186$ \\
\hline RRT (CVVHD)** & $26(12,2)$ & $19(17,8)$ & $7(6,6)$ & $p=0,01$ \\
\hline Creatinine max. (mg/dL) & $1,8 \pm 0,9$ & $1,9 \pm 0,9$ & $1,6 \pm 0,9$ & $p=0,001$ \\
\hline
\end{tabular}

\section{DISCUSSION}

The occurrence of AKI after TAVI procedures is one of most frequent complication with an incidence of $11-40 \%$. The AKI occurs as an independent predictor of mortality, increasing the risk more than four-fold in the TAVI population [6-9]. Moreover, kidney function impairment is very common issue in high risk elderly TAVI candidates and the fact that the CM exhibits nephrotoxic features in this population is beyond doubt $[10,11$, and 17].

However, there is a lack of evidence about the clinical relevance of CM-induced AKI (such as the influence on postoperative RRT), particularly in patients with CKD undergoing TAVI. Furthermore, the favorable timing of a dual CM exposition (CTA and intra-operative fluoroscopy) in high-risk elderly patients with compromised renal function is not clear. In this study, we aimed to find out if our CTA protocols with an intra-venous application of $\mathrm{CM}, 24 \mathrm{~h}$ prior to TA-TAVI, led to postoperative AKI and what the clinical impact was on elderly patients with CKD co morbidity undergoing TA-TAVI. Although, significantly more CM volume was applied peri-operatively in the CTA group compared to the TOE group, 
Does Preoperative Computed Tomography Angiography Lead to an Increased Incidence of Postoperative Acute Kidney Injury Following Transcatheter Aortic Valve Implantation in Patients with Impaired Renal Function?

we found that peri-operative additional intravenous application of $60 \mathrm{ml}$ Solutrast 370 for CTA examination did not lead to an increased postoperative AKI incidence in TATAVI patients. In this study, we specially focussed on the patient group with prior CKD. Surprisingly, we did not observed any association between the CTA group with CKD and increased postoperative AKI occurrence. In fact, we saw here a lower AKI incidence compared to the TOE-group with CKD.

The data on the risk of AKI requiring RRT after TA-TAVI in patients who received CM are limited. We were able to show that even in patients with CKD in our cohort, who received CTA with CM, required significantly lesser use of postoperative RRT compared to the TOEgroup with CKD. Nobody from the CTA-group died postoperatively due to AKI-related complications. These facts could be explainable by the baseline and perioperative characteristics of the cohorts. The TOE cohort includes older patients, higher preoperative serum creatinine level, longer operation time and more patients with congestive heart failure. Moreover, the postoperative bleeding volume was higher in the TOE group. All these features have been identified in other studies [6-9] as independent predictors of AKI after TAVI procedures. However, the CTA did not influence the TATAVI procedure in terms of the use of various prosthesis sizes. We measured at average bigger annulus via CTA but saw similar implanted prosthesis size in both groups.
Furthermore, the incidence of AKI after intraarterial administration of $\mathrm{CM}$ is up to threetimes higher than after intra-venous administration of $\mathrm{CM}[6,20$, and 21]. This might be another explanation to why postoperative AKI and its clinical complications occurred more frequently in the TOE group. We administrated on average $8 \mathrm{ml}$ more intraarterial CM for fluoroscopy in the TOE group, which was statistically significant [Fig.5]. Recent studies [12, 13] have shown, that not only high $\mathrm{CM}$ volumes but also a repetitive administration of $\mathrm{CM}$ within a short period of time $(<24 \mathrm{~h})$ correlate with increased risk of contrast-induced nephropathy.

We performed CTA examination on all patients $24 \mathrm{~h}$ before the TAVI procedure, independently of CKD co morbidity. Our strategy for TAVI immediately after CTA examination, with repetitive CM application within $24 \mathrm{~h}$, have been shown to be safe in terms of postoperative AKI, and cost-effective and useful in emergency TAVI cases. CTA-TAVI protocols described in the literature $[5,14-16]$ usually use large CM volumes up to $120 \mathrm{ml}$, to ensure the optimal exposure of the aorta and peripheral arteries. Accordingly, the next goal is the development of protocols with very low $\mathrm{CM}$ volumes to avoid contrast-induced AKI. The first steps in this field have been taken. The feasibility of 55 $\mathrm{ml}$ or even $20 \mathrm{ml} \mathrm{CM}$ volume CTA for TAVI has been tested $[18,19]$. For such protocols, we have limited data in terms of postoperative CMinduced AKI.

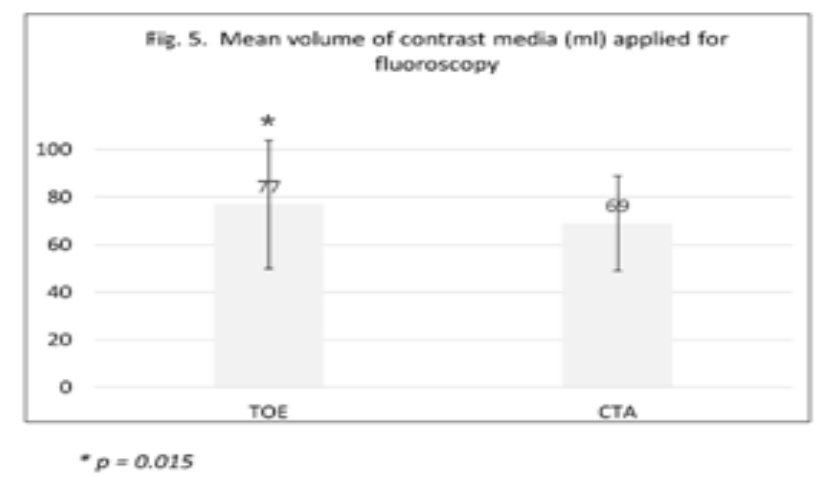

Fig5: Intraoperatively applied intra-arterial volume contrast media for fluoroscopy in both CTA and TOE groups.

\section{CONClusion}

We found that a CTA with $60 \mathrm{ml}$ CM application was a safe method prior to TATAVI, and did not trigger the post-procedural AKI occurrence. A consecutive and immediate (within $24 \mathrm{~h}$ ) intra-arterial application of CM does not influence the occurrence of the postoperative AKI, even in patients with CKD. The application of CM volumes up to $73 \mathrm{ml}$ during fluoroscopy is a safe course. Intra-arterial administration of $\mathrm{CM}$ in elderly patients who undergo TA-TAVI seems to be relevant in CM- 
Does Preoperative Computed Tomography Angiography Lead to an Increased Incidence of Postoperative Acute Kidney Injury Following Transcatheter Aortic Valve Implantation in Patients with Impaired Renal Function?

induced nephropathy, so that a reducing volume of CM application during fluoroscopy should be considered.

\section{LIMITATIONS}

This is an observational retrospective study and the evidence value provided in our study is inferior compared to randomized trials. This science has the usual flaws inherent in retrospective observational studies. The biggest flaw is that the addition of CTA with CM is not the only change in the practice between the groups. The procedure time of the groups has been changed. As this compares a service at one time point with another, there could be changes in the experience of the surgeons that might have influenced the results. Other professionals such as anaesthetists involved will also have gained more experience and could, therefore, have refined their skills at recognizing and treating renal issues around the time of TAVI. Another limitation is that the group studied is selected. This is only for CTA performed solely for patients assessed for TA-TAVI, which is a less common procedure than transfemoral TAVI, to which the results cannot necessarily be extrapolated.

\section{REFERENCES}

[1] Kempfert J, Van Linden A, Lehmkuhl L, et al.; Aortic annulus sizing: echocardiographic versus computed tomography derived measurements in comparison with direct surgical sizing.Eur J Cardiothorac Surg. 2012 Oct; 42(4):627-33.

[2] Hayashida K, Bouvier E, Lefèvre $\mathrm{T}$, et al; Impact of CT-guided valve sizing on postprocedural aortic regurgitation in transcatheter aortic valve implantation.EuroIntervention. 2012 Sep;8(5):546-55.

[3] Binder RK, Webb JG, Willson AB,Urena M etal.; The impact of integration of a multidetector computed tomography annulus area sizing algorithm on outcomes of transcatheter aortic valve replacement: a prospective, multicenter, controlled trial; J Am CollCardiol. 2013 Jul 30; 62(5):431-8.

[4] Husser O, Holzamer A, Resch M, et al.; Prosthesis sizing for transcatheter aortic valve implantation--comparison of three dimensional transesophageal echocardiography with multislice computed tomography.Int J Cardiol. 2013 Oct 9; 168(4):3431-8.

[5] Jilaihawi H, Kashif M, Fontana G, et al.; Crosssectional computed tomographic assessment improves accuracy of aortic annular sizing for transcatheter aortic valve replacement and reduces the incidence of paravalvular aortic regurgitation; J Am CollCardiol. 2012 Apr 3; 59(14):1275-86.

[6] Bagur R, Webb JG, Nietlispach F et al. Acute kidney injury following transcatheter aortic valve implantation: predictive factors, prognostic value, and comparison with surgical aortic valve replacement. Eur Heart J 2010; 31: 865-74.

[7] Elhmidi, Bleiziffer S, Piazza N, et al. Incidence and predictors of acute kidney injury in patients undergoing transcatheter aortic valve implantation. Am Heart J 2011; 161: 735-9.

[8] Ben-DorDvir D, Barbash IM, et al. Outcomes of Patients with Severe Aortic Stenosis at High Surgical Risk Evaluated in a Trial of Transcatheter Aortic Valve Implantation. Am J Cardiol. 2012 Oct 1; 110(7): 1008-14.

[9] Kong Yong G, Irish A.; Incidence, risk factors and prognosis of acute kidney injury after transcatheter aortic valve implantation. Nephrology 17 (2012) 445-451.

[10] Yamamoto M, Hayashida K, Mouillet G et al.; Renal function-based contrast dosing predicts acute kidney injury following transcatheter aortic valve implantation; JACC CardiovascInterv. 2013 May;6(5):479-86.

[11] Becker CR, Davidson C, Lameire N et al.; CIN Consensus Working Panel; High-risk situations and procedures; Am J Cardiol. 2006 Sep 18; 98(6A):37K-41K.

[12] Abujudeh HH, Gee MS, Kaewlai R; In emergency situations, should serum creatinine be checked in all patients before performing second contrast CT examinations within 24 hours? ; J Am CollRadiol. 2009 Apr; 6(4):268-73.

[13] Trivedi H1, Foley WD; Contrast-induced nephropathy after a second contrast exposure; Ren Fail. 2010; 32(7):796-801.

[14] Beeres M, Loch M, Schulz B et al.; Bolus timing in high-pitch CT angiography of the aorta; Eur J Radiol. 2013 Jun; 82(6):1028-33.

[15] Gurvitch R, Webb JG, Yuan R et al.; Aortic annulus diameter determination by multidetector computed tomography: reproducibility, applicability, and implications for transcatheter aortic valve implantation; JACC CardiovascInterv. 2011 Nov; 4(11):123 $5-45$.

[16] Nguyen G, Leipsic J; Cardiac computed tomography and computed tomography angiography in the evaluation of patients prior to transcatheter aortic valve implantation; CurrOpinCardiol. 2013 Sep; 28(5):497-504.

[17] Jochheim D, Schneider VS, Schwarz F et al.; Contrast-induced acute kidney injury after computed tomography prior to transcatheteraortic 
Does Preoperative Computed Tomography Angiography Lead to an Increased Incidence of Postoperative Acute Kidney Injury Following Transcatheter Aortic Valve Implantation in Patients with Impaired Renal Function?

valve implantation; Clin Radiol. 2014 Oct; 69(10):1034-8.

[18] Spagnolo P, Giglio M, Di Marco D et al.; Feasibility of ultra-low contrast 64-slice computed tomography angiography before transcatheter aortic valve implantation: a realworld experience; Eur Heart J Cardiovasc Imaging. 2016 Jan; 17(1):24-33.

[19] Azzalini L, Abbara S, Ghoshhajra BB; Ultralow contrast computed tomographic angiography (CTA) with $20-\mathrm{mL}$ total dose for transcatheter aortic valve implantation (TAVI) planning; J Comput Assist Tomogr. 2014 JanFeb; 38(1):105-9.
[20] Jan-Malte Sinning, AlexanderGhanem, HannahSteinhäuser et al. Renal Function as Predictor of Mortality in Patients after Percutaneous Transcatheter Aortic Valve Implantation. JACC: Cardiovascular InterventionsVolume 3, Issue 11, November 20 10, Pages 1141-1149

[21] Saia F, Ciuca C, Taglieri N et al. Acute kidney injury following transcatheter aortic valve implantation: incidence, predictors and clinical outcome.Int J Cardiol. 2013 Sep 30; 168(2):10 34-40.

Citation: Dritan Useini, Blerta Beluli, et.al. Does Preoperative Computed Tomography Angiography Lead to an Increased Incidence of Postoperative Acute Kidney Injury Following Transcatheter Aortic Valve Implantation in Patients With Impaired RenalFunction?. ARC Journal of Surgery.2019; 5(4):1-7. DOI: http://dx.doi.org/10.20431/2455-572X.0504001

Copyright: (C) 2019 Authors. This is an open-access article distributed under the terms of the Creative Commons Attribution License, which permits unrestricted use, distribution, and reproduction in any medium, provided the original author and source are credited. 